\title{
Modeling and Analysis of Harmonic Spurs in DLL-Based Frequency Synthesizers
}

\author{
Amin Ojani, Behzad Mesgarzadeh and Atila Alvandpour
}

\section{Linköping University Post Print}

\section{Tweet}

N.B.: When citing this work, cite the original article.

Amin Ojani, Behzad Mesgarzadeh and Atila Alvandpour, Modeling and Analysis of Harmonic Spurs in DLL-Based Frequency Synthesizers, 2014, IEEE Transactions on Circuits and Systems Part 1: Regular Papers, (61), 11, 3075-3084.

\section{http://dx.doi.org/10.1109/TCSI.2014.2321188}

C2014 IEEE. Personal use of this material is permitted. However, permission to reprint/republish this material for advertising or promotional purposes or for creating new collective works for resale or redistribution to servers or lists, or to reuse any copyrighted component of this work in other works must be obtained from the IEEE.

\section{http://ieeexplore.ieee.org/}

Postprint available at: Linköping University Electronic Press

http://urn.kb.se/resolve?urn=urn:nbn:se:liu:diva-110392 


\title{
Modeling and Analysis of Harmonic Spurs in DLL-Based Frequency Synthesizers
}

\author{
Amin Ojani, Student Member, IEEE, Behzad Mesgarzadeh, Member, IEEE, and Atila Alvandpour, \\ Senior Member, IEEE
}

\begin{abstract}
Periodic jitter raises the harmonic spurs at frequency synthesizer output spectrum, down-converting the outof-band interferers into the desired band and corrupting the wanted signal. This paper proposes a comprehensive behavioral model for spur characterization of edge-combining delay-locked loop (DLL)-based synthesizers, which includes the effects of delay mismatch, static phase error (SPE), and duty cycle distortion (DCD). Based on the proposed model and utilizing Fourier series representation of DLL output phases, an analytical model which formulates the synthesizer spur-to-carrier ratio (SCR) is developed. Moreover, from statistical analysis of the analytical derivations, a closed-form expression for SCR is obtained, from which a spur-aware synthesizer design flow is proposed. Employing this flow and without Monte Carlo (MC) method, one can determine the required stage-delay standard deviation (SD) of a DLL-based synthesizer, at which a certain spurious performance demanded by a target wireless standard is satisfied. A design example is presented which utilizes the proposed design flow to fulfill the SCR requirement of $\mathbf{- 4 5} \mathrm{dBc}$ for WiMedia-UWB standard. Transistor-level MC simulation of the synthesizer SCR for a standard 65-nm CMOS implementation exhibits good compliance with analytical models and predictions.
\end{abstract}

Index Terms - delay mismatch, DLL, duty cycle distortion, edge-combiner, frequency synthesizer, harmonic spur, periodic jitter, static phase error.

\section{INTRODUCTION}

$\mathrm{D}$ ELAY-LOCKED loop (DLL)-based frequency multipliers have expanded their applications during the last decades into wireless communication systems [1]-[8]. Essential DLL characteristics such as the relatively wide loop-bandwidth, fast lock-in time, limited-accumulative jitter, and first-order stability, indicate the potentials of DLL-based frequency generation schemes [9]. However, designing such systems for $\mathrm{RF}$ applications, where carrier signals of certain spectral purity must be provided, has its own challenges. Since the accumulated random jitter within the delay line is reset back to zero at every reference clock edge, edge-combining DLLbased synthesizers exhibit a flat phase noise profile [1], [4]. On the other hand, as the DLL is locked to the reference period and the carrier is generated by combining the equallyspaced DLL edges, any misalignment of those edges result in periodic jitter and raises the reference harmonic spurs at synthesizer output spectrum, down-converting the out-of-band interferers into the desired band. One of the major contributors to the level of spurious tones in such synthesizers is the duty cycle distortion (DCD) of the reference clock as well as the internal DLL phases. In addition, the static phase error (SPE) between the reference and feedback signals caused by up/down pulsewidth and current imbalances in phase detector (PD) and charge pump (CP) respectively, deteriorates the spurious performance. Moreover, delay mismatches among delay stages in the delay line, made by the local manufacturing imperfections, will also lead to harmonic spurs. Implied by the matching properties of MOS devices [10], to minimize the delay mismatch between delay stages, large-area devices should be utilized. Due to stochastic nature of the mismatch, statistical simulations such as Monte Carlo (MC) with large number of samples should be performed for accurate prediction of the synthesizer spurious performance. However, for such complex and limited-bandwidth feedback systems which require a large settling time for each MC sample, circuit-level MC becomes extremely cumbersome. Now consider that to satisfy a certain requirement on harmonic spurs and to avoid over-sizing the delay stages which leads to area and speed penalties, optimal device sizes need to be found by iteratively improving the circuit parameters and performing a new set of circuit-level MC to verify the obtained performance. Consequently, to accelerate the design procedure, it is crucial to develop an accurate behavioral model of the spur characteristics of DLL-based synthesizers, which includes all the main sources of periodic jitter. A thorough analytical model which formulates the spurious performance in terms of DCD, SPE and delay standard deviation (SD) is hence of great interest. A few works [4], [11]-[12] have studied the spur characteristics of edgecombining synthesizers, from which [4] and [11] limit their analysis solely to the effect of SPE. In [12], the effect of delay mismatch is also included, but the model is customized only for rising edge combiners (EC) and does not consider the effect of DCD on synthesizer's harmonic spurs. When both the clock edges of the DLL phases are used by the EC, the duty cycle variations of those phases will generate a periodicity. This implies that besides SPE and delay SD, the levels of harmonic spurs become also a function of DCD, which is not modeled in [12]. Therefore, it cannot be employed as a general model of edge-combing synthesizers for spur characterization.

In this work, we propose a comprehensive behavioral model of edge-combining DLL-based synthesizers, which includes 


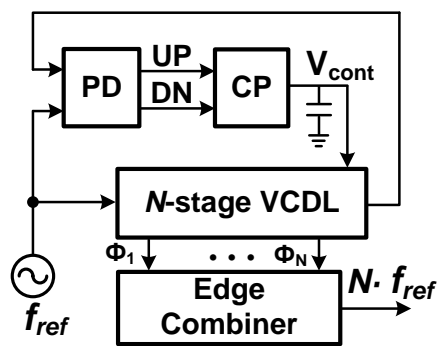

(a)

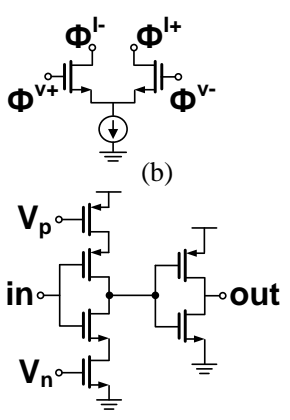

(c)
Fig. 1. (a) Block diagram of a DLL-based EC frequency synthesizer, (b) a V-I converter stage of EC, and (c) an active implementation of the delay stage.

the impact of DCD as well as the SPE and delay mismatch, on synthesizer spurious performance. Utilizing this model and the Fourier series representation of multiphase DLL outputs, an analytical model is developed, formulating the synthesizer spur-to-carrier ratio (SCR). We further expand our analysis by aid of statistical methods, to obtain a closed-form expression for mean SCR and its probability density function (PDF). Utilizing our closed-form formula, we propose a spur-aware synthesizer design methodology. Employing this design flow and without MC simulations, the required delay SD which satisfies a certain SCR requirement, can be accurately estimated. Based on the calculated delay SD, one can design the corresponding delay stage circuit.

The paper is organized as follows. Section II proposes a behavioral model of edge-combining DLL-based synthesizers. In Section III, an analytical model is derived from the Fourier series representation of multiphase DLL outputs. Statistical analysis which results in a close-form expression for synthesizer's spur levels is presented in Section IV. In Section $\mathrm{V}$, a spur-aware synthesizer design methodology is proposed which is utilized in the design example of Section VI to find the required stage-delay $\mathrm{SD}$ which satisfies the spurious performance of a WiMedia ultra-wideband (UWB) standard. Finally, the paper is concluded in Section VII.

\section{BEHAVIORAL MODEL OF DLL-BASED SYNTHESIZERS}

Consider the edge-combining DLL-based synthesizer diagram shown in Fig. 1(a), where the system is locked to a reference clock frequency $f_{\text {ref }}$ and generates a carrier at $f_{c}$ $=N \times f_{\text {ref }}$ by combining $N$ evenly-spaced DLL phases. To characterize the spurious performance, we propose a behavioral feedforward model of the in-lock synthesizer, which considers the effects of the delay mismatch, DCD, and SPE. Note that the model is developed based on a risingfalling current summation EC [1] which employs voltage-tocurrent (V-I) converter stages shown in Fig. 1(b). The architecture requires a voltage-controlled delay line (VCDL) with odd number of delay stages $N$. Nevertheless, the model is generic and can be applied to synthesizers of other types of DLL/EC configurations. To model the delay mismatch, we assume that the delay of each stage is a random variable of normal distribution. Note that in active implementations of the delay stage such as the one in Fig. 1(c), pull-up and pull-down parts responsible for delaying the falling and rising edges

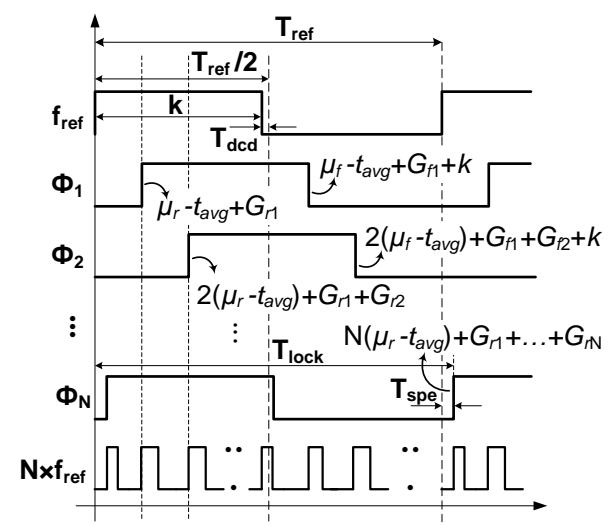

Fig. 2. Waveform representation of $N$-stage DLL-based synthesizer including DCD, SPE and delay mismatch effects.

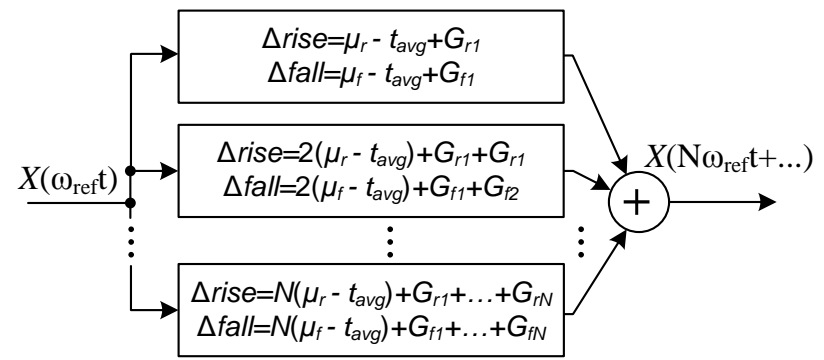

Fig. 3. Proposed Time-domain feedforward model of in-lock DLL-based EC synthesizer.

respectively, are of different physical properties. This implies that the rising edge time delay $\Delta t_{d, r}$ and falling edge time delay $\Delta t_{d, f}$, experience different mismatch profiles. Therefore, we model them as two independent Gaussian random variables

$$
\Delta t_{d, r} \sim \mathrm{N}\left(\mu_{r}, \sigma_{r}^{2}\right), \Delta t_{d, f} \sim \mathrm{N}\left(\mu_{f}, \sigma_{f}^{2}\right)
$$

where $\mu_{r}, \sigma_{r}^{2}, \mu_{f}$, and $\sigma_{f}^{2}$ are the mean and variance of $\Delta t_{d, r}$ and $\Delta t_{d, f}$, respectively. A waveform representation of the proposed synthesizer model is illustrated in Fig. 2. To determine $\Delta t_{d, r}$ and $\Delta t_{d, f}$, we assume that the DLL is locked to the reference clock period $T_{r e f}$, but with a time-domain SPE of $T_{\text {spe. }}$ So, the total VCDL delay length would be

$$
T_{V C D L}=T_{\text {lock }}=T_{\text {ref }}+T_{\text {spe }} .
$$

We assume that the mean of the rising and falling edge delays are equal. So, for the in-lock DLL with an $N$-stage VCDL,

$$
\mu_{r}=\mu_{f}=\mu=T_{\text {lock }} / N \text {. }
$$

Hence, $\Delta t_{d, r}$ and $\Delta t_{d, f}$ for the $m^{\text {th }}$ delay stage, are represented as

$$
\Delta t_{d, r m}=\frac{T_{l o c k}}{N}-t_{\text {avg }}+G_{r m}, \Delta t_{d, f m}=\frac{T_{l o c k}}{N}-t_{a v g}+G_{f m}
$$

where $m \in[1, N]$, and $G_{r m}$ and $G_{f m}$ are independent zero-mean Gaussian variables, representing the delay mismatch of rising and falling edges, respectively, and extracted from $\Delta t_{d, r}$ and $\Delta t_{d, f}$ to simplify the analysis. So,

$$
G_{r 1}, \ldots, G_{r N} \sim \mathrm{N}\left(0, \sigma_{r}^{2}\right), G_{f 1}, \ldots, G_{f N} \sim \mathrm{N}\left(0, \sigma_{f}^{2}\right) .
$$

Note that $t_{\text {avg }}$ is an offset delay which is applied by the lockedloop to all the stages, such that despite the delay mismatch, the total VCDL delay length maintains equal to $T_{\text {lock. }}$ In our feedforward model, this actually models the mismatchaveraging characteristic of the in-lock DLL. To calculate $t_{\text {avg }}$, we assume a rising-edge-locked system. So,

$$
T_{\text {lock }}=\sum_{m=1}^{N} \Delta t_{d, r m} .
$$


In addition, we can write from (4) that

$$
\sum_{m=1}^{N} \Delta t_{d, r m}=T_{\text {lock }}-N t_{\text {avg }}+\sum_{m=1}^{N} G_{r m} .
$$

Now, from (6) and (7), $t_{\text {avg }}$ is found as a random variable that consists of the averaged sum of $N$ random variables that is

$$
t_{\text {avg }}=\frac{1}{N} \sum_{m=1}^{N} G_{r m} \sim \mathrm{N}\left(0, \sigma_{r}^{2} / N\right) .
$$

Note that parameter $k$ (Fig. 2) models the input clock pulsewidth. So, the time-domain DCD value $T_{d c d}$ is written as

$$
T_{d c d}=\left(T_{r e f} / 2\right)-k
$$

Also note that as mentioned previously, apart from the DCD of the reference clock, the duty cycle is also distorted due to the inherent differences in physical properties of pull-up and pulldown devices. By linearity, it can be shown that the so-called internal duty cycle distortion (IDCD) within a delay stage follows a normal distribution defined as

$$
\mathrm{IDCD}=\Delta t_{d, r}-\Delta t_{d, f} \sim \mathrm{N}\left(\mu_{r}-\mu_{f}, \sigma_{r}^{2}+\sigma_{f}^{2}\right) .
$$

The above expression implies that an accurate ratio sizing between the pull-up and pull-down devices, as assumed in (3), can only eliminate the mean of IDCD, demonstrating that the inter-stage DCD is correlated to the delay mismatch.

Fig. 3 depicts the time-domain block diagram of our feedforward model where the combined output, containing the fundamental as well as reference harmonic components, is generated by summation of the time-shifted versions of the rising and falling reference clock edges. The model is implemented in MATLAB and the SCR at synthesizer's output spectrum for each MC sample (of randomized $G_{r}$ and $G_{f}$ values) is calculated by taking the fast Fourier transform (FFT) of the output signal. This model is employed as a reference for spurious performance analysis in upcoming sections.

\section{Analytical Modeling Using Fourier SERIES}

Based on the introduced behavioral model in Section II, we develop an analytical model which formulates the synthesizer spurious performance in terms of DCD, SPE and delay SD. Containing the magnitude, phase and frequency information of periodic signals, Fourier series are efficient tools for analyzing the square wave multiphase DLL outputs. In Appendix A, we derive the Fourier series of the EC output signal from those of the DLL output phases according to Fig. 2. As a result, $a_{n}$ Fourier coefficient of the synthesizer output is represented as

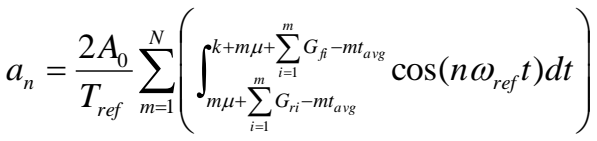

$$
\begin{aligned}
& =\frac{2 A_{0}}{n \pi} \sum_{m=1}^{N}\left[\sin \frac{n \pi}{T_{r e f}}\left(k+X_{f m}-X_{r m}\right)\right. \\
& \left.\cos \frac{n \pi}{T_{r e f}}\left(\frac{2 m\left(T_{r e f}+T_{s p e}\right)}{N}+k+X_{f m}+X_{r m}\right)\right]
\end{aligned}
$$

where $A_{0}, \omega_{r e f}$, and $n$ are the amplitude, angular frequency and (integer) harmonic index of the reference clock, respectively, and $X_{f m}$ and $X_{r m}$ are random variables defined as

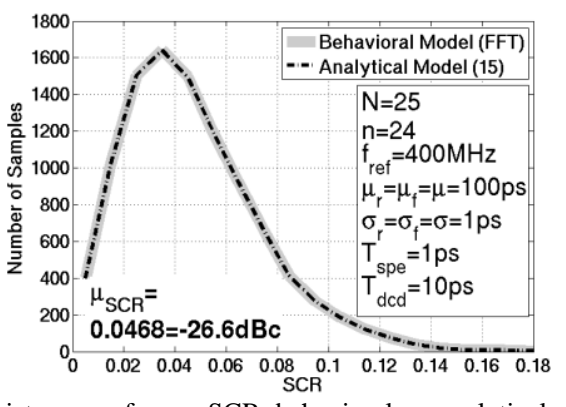

Fig. 4. MC histogram of mean SCR; behavioral vs. analytical model (15).

$$
X_{f m}=-\frac{m}{N} \sum_{i=1}^{N} G_{r i}+\sum_{i=1}^{m} G_{f i}, X_{r m}=-\frac{m}{N} \sum_{i=1}^{N} G_{r i}+\sum_{i=1}^{m} G_{r i} .
$$

Similarly, for $b_{n}$ coefficient of the EC output, we can write

$$
\begin{aligned}
b_{n}=\frac{2 A_{0}}{n \pi} \sum_{m=1}^{N} & {\left[\sin \frac{n \pi}{T_{r e f}}\left(k+X_{f i n}-X_{r m}\right)\right.} \\
& \left.\sin \frac{n \pi}{T_{r e f}}\left(\frac{2 m\left(T_{r e f}+T_{s p e}\right)}{N}+k+X_{f m}+X_{r m}\right)\right]
\end{aligned}
$$

We can now find the magnitude of the vector $\left(a_{n}, b_{n}\right)$ which represents the output spur magnitude for a given harmonic $n$ located at $f_{s}=n \times f_{\text {ref. }}$. Thus,

$$
S_{n}=\left|a_{n}+j b_{n}\right|=\sqrt{a_{n}^{2}+b_{n}^{2}} .
$$

The synthesizer's SCR magnitude is therefore expressed as

$$
S C R_{n}=\frac{S_{n}}{C}=\frac{\left|a_{n}+j b_{n}\right|}{\left|a_{N}+j b_{N}\right|}=\frac{\sqrt{a_{n}^{2}+b_{n}^{2}}}{\sqrt{a_{N}^{2}+b_{N}^{2}}}
$$

where $C$ is the fundamental tone (carrier) magnitude, located at $f_{c}$ and calculated from (14) for $n=N$. To verify the derived analytical expression, Fig. 4 compares the MC histogram of the SCR distribution obtained from our reference behavioral model, with that of attained from the analytical model (15). Note that for the simulations presented in the current section as well as in Section IV, we utilize the following parameters, unless otherwise specified. The reference clock is $f_{\text {ref }}=400$ $\mathrm{MHz}$ and the VCDL has $N=25$ stages, generating a carrier at $f_{c}=25 \times 400 \mathrm{MHz}$. The SCR is simulated for the largest spur located at $f_{s}=f_{c}-f_{\text {ref }}(n=N-1)$. Furthermore, $T_{d c d}$ and $T_{s p e}$ are assumed to be $10 \%$ and $1 \%$ of the mean stage-delay $\mu=100 \mathrm{ps}$, which are $10 \mathrm{ps}$ and $1 \mathrm{ps}$, respectively. Also, the numerical rising and falling edge delay SD values are $\sigma_{r} / \mu=\sigma_{f} / \mu=1 \%=$ 1 ps. Now, to acquire a better insight into (15), we depict the dependence of the mean SCR, as a function of the delay SD, DCD, SPE, and $N$. It can be observed from Fig. 5(a) and (b) that the SCR is solely defined by the delay SD for small values of DCD and SPE, respectively, and as these parameters get larger, the SCR becomes a function of DCD and SPE as well. Therefore, it is crucial to maintain DCD and SPE sufficiently small, so that we can efficiently get benefit from reducing the delay SD. On the other hand, for a given delay SD, overimproving the SPE and DCD, might not have a significant contribution in lowering the level of spurs, as there exists a lower bound on SCR defined by the delay SD. For this specific example, it can be observed from Fig. 5(a) and (b), that even with a quite small delay SD of $\sigma / \mu=0.2 \%$, pushing $T_{d c d}$ and $T_{\text {spe }}$ below $10 \mathrm{ps}$ and $1 \mathrm{ps}$, respectively, would not improve 


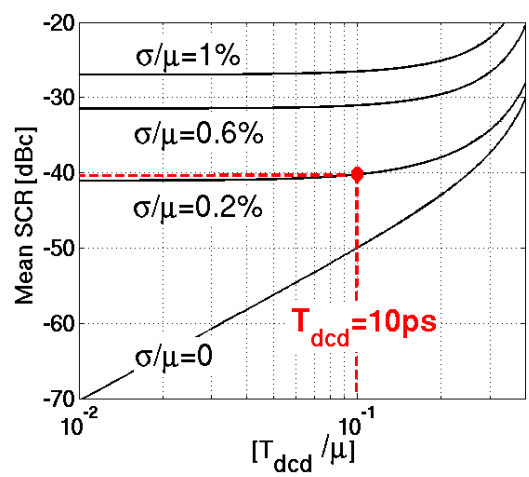

(a)

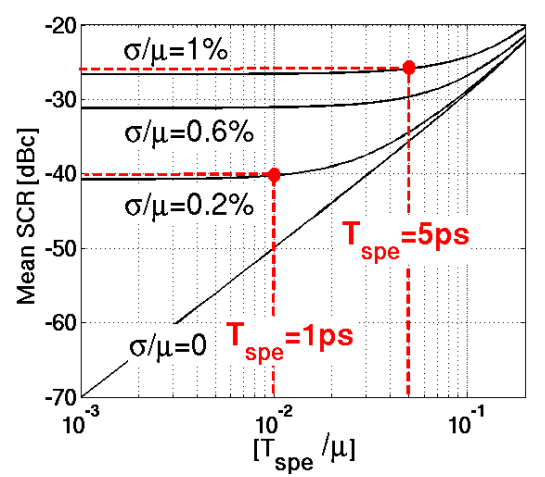

(b)

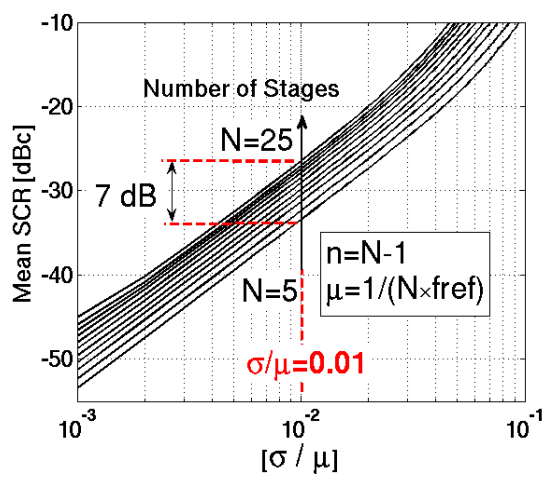

(c)

Fig. 5. Simulated mean SCR of the synthesizer from the analytical expression (15), as a function of normalized: (a) DCD, (b) SPE, and (c) stage-delay SD.

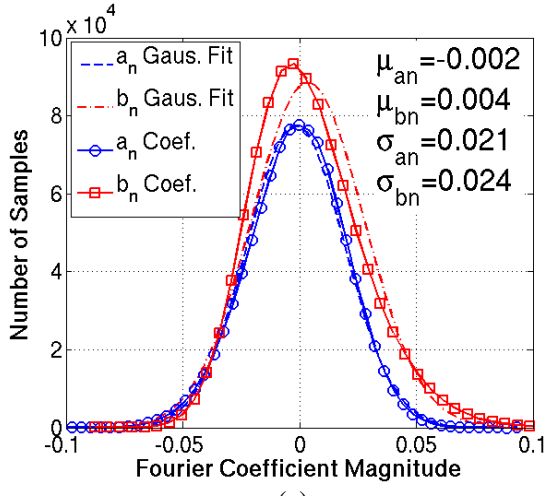

(a)

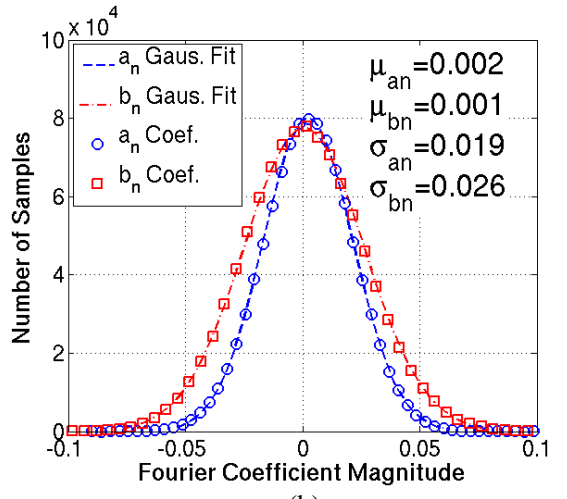

(b)

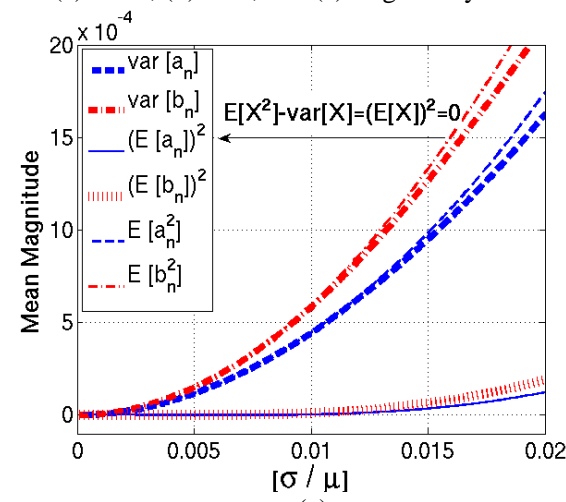

(c)

Fig. 6. Graphical test of criteria (16) for $a_{n}$ and $b_{n}(n=N-1)$ : (a) in-lock normality, (b) open-loop normality, and (c) zero-mean and equal-variance.

SCR much. Also for the case of $\sigma / \mu=1 \%$, it can be noted from Fig. 5(b) that by pushing $T_{s p e}$ below 5 ps, we do not gain any further spur suppression. Fig. 5(c) illustrates how the SCR changes as the delay SD varies for different $N$. As can be observed, for fixed DCD and SPE, and a given normalized delay SD of $\sigma / \mu=1 \%$, increasing $N$ from 5 to 25 to achieve a higher carrier frequency of 5 times, results in about $7 \mathrm{~dB}$ degradation in spurious performance, even though the delay $\mathrm{SD}$ is scaled with $N$ to keep $\sigma / \mu$ constant.

\section{CLOSED-Form EXPRESSION FOR SYNTHESIZER SCR}

In this section, we further expand our analytical model from the previous section by utilizing moment methods and Taylor series approximations, and obtain closed-from expressions for the mean and PDF of SCR at synthesizer output spectrum. As a result, we can estimate the synthesizer spurious performance without performing MC. In part A, we analyze the random variable $S_{n}$ (14), i.e. the harmonic spur magnitude, and show that it can be approximated as a Rayleigh random variable. To estimate the SCR distribution (15), we show in part B that the random variable $C$, i.e. the carrier magnitude, can be approximated as a constant value that is equal to its mean.

\section{A. Magnitude of Spurious Tones}

Provided that the spur Fourier coefficients $a_{n}$ and $b_{n}$ at the EC output represented by (11) and (13), are two independently and identically distributed (iid) Gaussian random variables of zero mean and equal variances $\sigma_{\text {cof }}^{2}$, i.e.,

$$
a_{n} \sim \mathrm{N}\left(0, \sigma_{c o f}^{2}\right), \quad b_{n} \sim \mathrm{N}\left(0, \sigma_{c o f}^{2}\right) \quad n \neq N,
$$

then the spur magnitude $S_{n}=\sqrt{a_{n}^{2}+b_{n}^{2}}$ will be a Rayleigh random variable [13]. Assuming $a_{n}$ and $b_{n}$ are independent, if the criteria of (16) are satisfied, we can efficiently characterize the harmonic spurs of the synthesizer, using the properties of Rayleigh distribution. To investigate (16), we start from the normality test. Statistical approaches involve calculation of skewness and kurtosis of the coefficients. In this work, we use graphical approach which has less complexity and works well for our approximation. Using (11) and (13), the MC histograms of the output spur Fourier coefficients are plotted in Fig. 6(a), indicating that $a_{n}$ fits well within its corresponding Gaussian dashed curve. The observed skew in $b_{n}$ histogram is due to the rising-edge-delay mismatch averaging characteristic of the DLL, whose averaged value $t_{\text {avg }}$ is applied to both the rising and falling edge delays, according to (4). This can be verified by plotting the histograms in Fig. 6(b), for an openloop case $\left(t_{\text {avg }}=0\right)$, where no skew on $b_{n}$ is observed. Nevertheless, as the skew in the closed-loop case is small, $b_{n}$ coefficient is also considered as a normal variable.

To evaluate the Fourier coefficients of the output spur as a function of delay SD, we utilize the variance formula of

$$
\operatorname{var}[X]=E\left[X^{2}\right]-(E[X])^{2}
$$

in which $E[X]$ is the expectation operator. It can be inspected form Fig. 6(c), that for small delay SDs, the variance and second moment $E\left[X^{2}\right]$ are equal for each coefficient, indicating that the corresponding mean values $E[X]$ are sufficiently small to be considered as zero. In addition, it can be noted that the variance of $a_{n}$ and $b_{n}$ are almost identical for small SDs. This implies that the criteria of (16) can be considered as valid as long as the delay SD maintains sufficiently small. This is in fact the case in the context of frequency synthesis with stringent requirements on spur levels. Therefore, we 


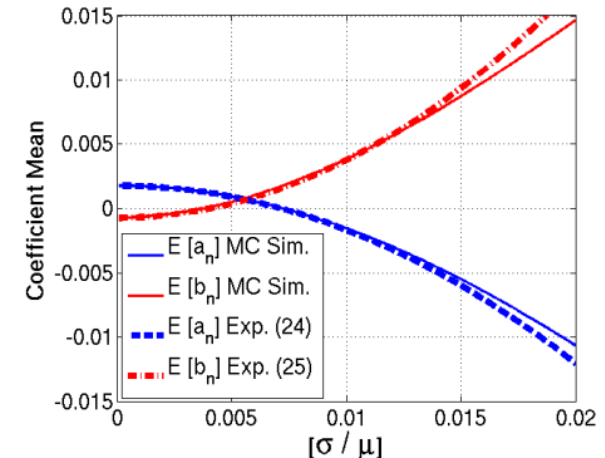

(a)

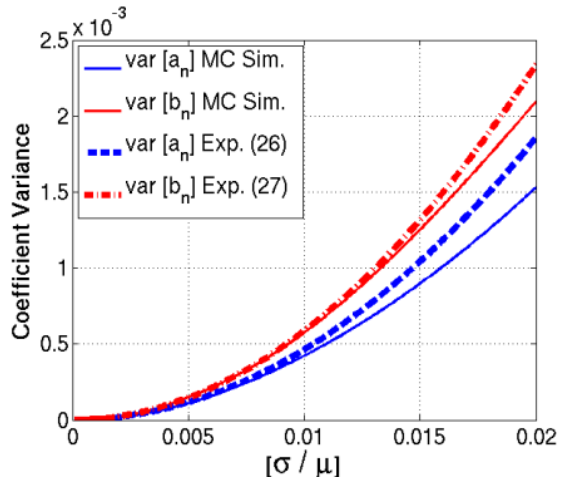

(b)

Fig. 7. Predicted and simulated (a) mean, and (b) variance of the Fourier coefficients $a_{n}$ and $b_{n}$ of the output spur, as a function of normalized delay SD.

approximate the magnitude of the output spurs $S_{n}$ as a Rayleigh random variable with $\mathrm{PDF}$ and mean that are expressed respectively as

$$
\begin{gathered}
p_{X}(x)= \begin{cases}\left(x / \sigma_{\text {cof }}^{2}\right) \exp \left(-x^{2} / 2 \sigma_{\text {cof }}^{2}\right) & x \geq 0 \\
0 & x<0\end{cases} \\
E\left[S_{n}\right]=\sigma_{\text {cof }} \sqrt{\pi / 2}
\end{gathered}
$$

where $\sigma_{\text {cof }}^{2}=\operatorname{var}\left[a_{n}\right] \approx \operatorname{var}\left[b_{n}\right]$. To determine (18) and (19), we need to find the first and second moments of the output Fourier coefficients, and calculate their variance from (17). So, we expand the expression for $a_{n}$ coefficient in (11) as

$$
\begin{aligned}
a_{n}=\frac{A_{n}}{2} \sum_{m=1}^{N}[ & \alpha_{m} \kappa_{s}\left(\cos \left(2 \lambda X_{f m}\right)+\cos \left(2 \lambda X_{r m}\right)\right) \\
& +\alpha_{m} \kappa_{c}\left(\sin \left(2 \lambda X_{f m}\right)-\sin \left(2 \lambda X_{r m}\right)\right) \\
& -\beta_{m} \kappa_{s}\left(\sin \left(2 \lambda X_{f m}\right)+\sin \left(2 \lambda X_{r m}\right)\right) \\
& \left.-\beta_{m} \kappa_{c}\left(\cos \left(2 \lambda X_{r m}\right)-\cos \left(2 \lambda X_{f m}\right)\right)\right]
\end{aligned}
$$

where

$$
\begin{gathered}
A_{n}=\frac{2 A_{0}}{n \pi}, \lambda=\frac{n \pi}{T_{r e f}}, \kappa_{s}=\sin \frac{k n \pi}{T_{r e f}}, \text { and } \kappa_{c}=\cos \frac{k n \pi}{T_{r e f}} \\
\alpha_{m}=\cos \left(\frac{n \pi}{T_{r e f}}\left[\frac{2 m}{N}\left(T_{r e f}+T_{s p e}\right)+k\right]\right) \\
\beta_{m}=\sin \left(\frac{n \pi}{T_{r e f}}\left[\frac{2 m}{N}\left(T_{r e f}+T_{s p e}\right)+k\right]\right)
\end{gathered}
$$

In Appendix B, we first simplify (20) by utilizing the first and second-order Taylor series approximation of sine and cosine respectively, and then obtain the mean of $a_{n}$ by finding its first moment. This can be similarly done for $b_{n}$. As a result,

$$
\begin{array}{r}
E\left[a_{n}\right]=A_{n} \sum_{m=1}^{N}\left\{\alpha_{m} \kappa_{s}-\alpha_{m} \kappa_{s} \lambda^{2} m\left(\sigma_{f}^{2}+\sigma_{r}^{2}\right)\right. \\
\left.-\beta_{m} \kappa_{c} \lambda^{2} m\left(\sigma_{f}^{2}+\sigma_{r}^{2}\left(\frac{2 m}{N}-1\right)\right)\right\} \\
E\left[b_{n}\right]=A_{n} \sum_{m=1}^{N}\left\{\beta_{m} \kappa_{s}-\beta_{m} \kappa_{s} \lambda^{2} m\left(\sigma_{f}^{2}+\sigma_{r}^{2}\right)\right. \\
\left.+\alpha_{m} \kappa_{c} \lambda^{2} m\left(\sigma_{f}^{2}+\sigma_{r}^{2}\left(\frac{2 m}{N}-1\right)\right)\right\} .
\end{array}
$$

As also discussed in Appendix $\mathrm{B}$, to find $a_{n}$ variance, we determine $\left(E\left[a_{n}\right]\right)^{2}$ and $E\left[a_{n}{ }^{2}\right]$, and substitute them in (17) to get

$$
\begin{gathered}
\operatorname{var}\left[a_{n}\right]=\left(A_{n} \lambda\right)^{2}\left\{\kappa_{c}^{2}\left(\sigma_{f}^{2}+\sigma_{r}^{2}\right)\left(\sum_{m=1}^{N} m \alpha_{m}^{2}+2 \sum_{m=1}^{N-1}\left(m \alpha_{m} \sum_{i=m+1}^{N} \alpha_{i}\right)\right)\right. \\
+\kappa_{s}^{2}\left(\sigma_{f}^{2}+\sigma_{r}^{2}\right)\left(\sum_{m=1}^{N} m \beta_{m}^{2}+2 \sum_{m=1}^{N-1}\left(m \beta_{m} \sum_{i=m+1}^{N} \beta_{i}\right)\right) \\
-2 \kappa_{s} \kappa_{c} \sum_{m=1}^{N} \alpha_{m}\left(\sum_{i=1}^{m} i \beta_{i}\left[\sigma_{f}^{2}+\left(\frac{2 m}{N}-1\right) \sigma_{r}^{2}\right]\right. \\
\left.\left.+m \sum_{i=m+1}^{N} \beta_{i}\left[\sigma_{f}^{2}+\left(\frac{2 i}{N}-1\right) \sigma_{r}^{2}\right]\right)\right\}
\end{gathered}
$$

Similarly for $b_{n}$ coefficient, the variance is derived as

$$
\begin{aligned}
& \operatorname{var}\left[b_{n}\right]=\left(A_{n} \lambda\right)^{2}\left\{\kappa_{c}^{2}\left(\sigma_{f}^{2}+\sigma_{r}^{2}\right)\left(\sum_{m=1}^{N} m \beta_{m}^{2}+2 \sum_{m=1}^{N-1}\left(m \beta_{m} \sum_{i=m+1}^{N} \beta_{i}\right)\right)\right. \\
&+ \kappa_{s}^{2}\left(\sigma_{f}^{2}+\sigma_{r}^{2}\right)\left(\sum_{m=1}^{N} m \alpha_{m}^{2}+2 \sum_{m=1}^{N-1}\left(m \alpha_{m} \sum_{i=m+1}^{N} \alpha_{i}\right)\right) \\
&+2 \kappa_{s} \kappa_{c} \sum_{m=1}^{N} \beta_{m}\left(\sum_{i=1}^{m} i \alpha_{i}\left[\sigma_{f}^{2}+\left(\frac{2 m}{N}-1\right) \sigma_{r}^{2}\right]\right. \\
&\left.\left.+m \sum_{i=m+1}^{N} \alpha_{i}\left[\sigma_{f}^{2}+\left(\frac{2 i}{N}-1\right) \sigma_{r}^{2}\right]\right)\right\}
\end{aligned}
$$

The value of $\sigma_{\text {cof }}^{2}$ in (18) and (19) can be approximated by the averaged sum of (26) and (27) as

$$
\begin{aligned}
\sigma_{\text {cof }}^{2} \approx \frac{\operatorname{var}\left[a_{n}\right]+\operatorname{var}\left[b_{n}\right]}{2}=\left(\frac{2 A_{0}}{T_{r e f}}\right)^{2}\left(\sigma_{f}^{2}+\sigma_{r}^{2}\right) \\
\times\left(\frac{N(N+1)}{4}+\sum_{m=1}^{N-1} m\left(\alpha_{m} \sum_{i=m+1}^{N} \alpha_{i}+\beta_{m} \sum_{i=m+1}^{N} \beta_{i}\right)\right) .
\end{aligned}
$$

To verify the derived mean and variance expressions, we plot them as dashed lines in Fig. 7(a) and (b), respectively, and compare them with those corresponding values attained from MC of our analytical model (15). As can be observed, for small delay SDs, the derived expressions match the simulations. For large delay SDs, the accuracy degrades due to the low-order Taylor series which was utilized to approximate $a_{n}$ and $b_{n}$. Nonetheless, higher order Taylor approximation which results in complex derivations would not be necessary, because as mentioned before, the large values of SDs are in fact avoided due to the stringent requirements on synthesizer's output spur levels.

\section{B. Magnitude of Spur-to-Carrier Ratio (SCR)}

As indicated previously by (15), the synthesizer SCR is the 


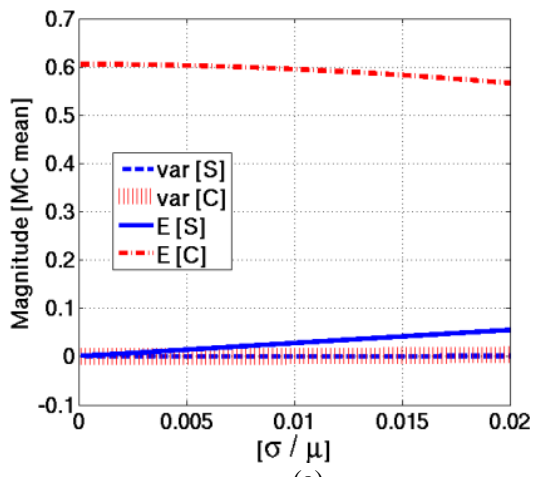

(a)

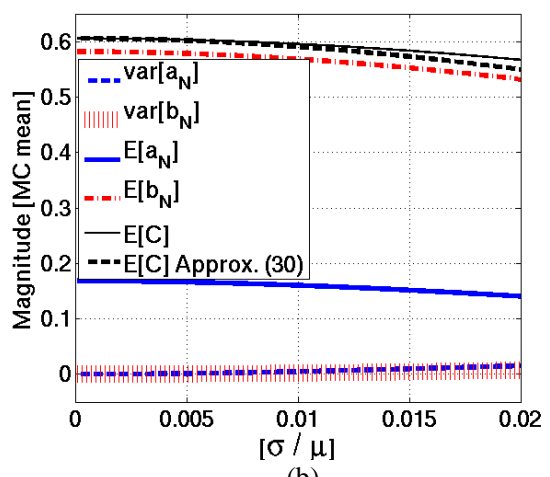

(b)

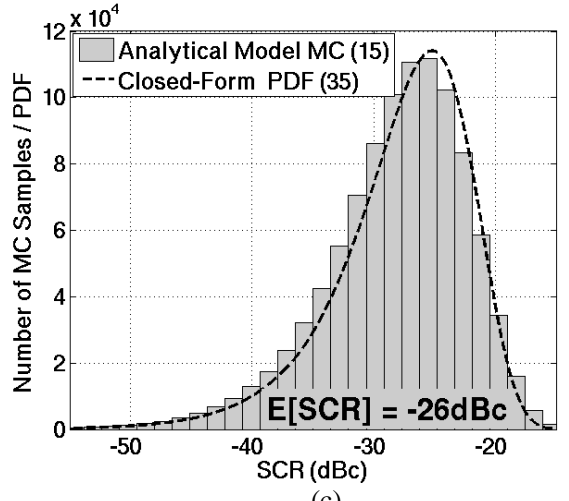

(c)

Fig. 8. (a) Simulated mean and variance of the carrier and spur magnitudes $\left(C\right.$ and $\left.S_{n}\right)$ as a function of normalized delay SD, (b) simulated mean and variance of the carrier's Fourier coefficients $a_{N}$ and $b_{N}$ as a function of normalized delay SD, and (c) simulated and predicted synthesizer SCR for the spur at $f_{s}=f_{c}-f_{r e f}$.

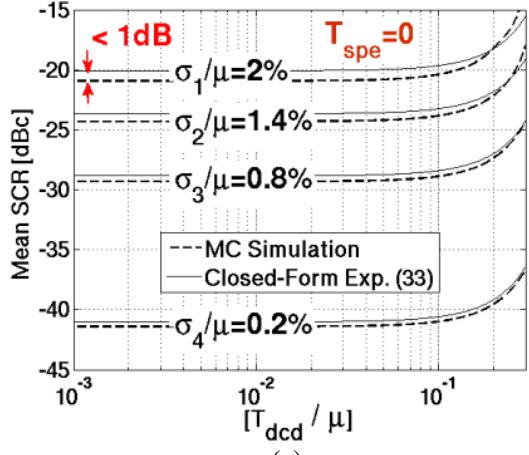

(a)

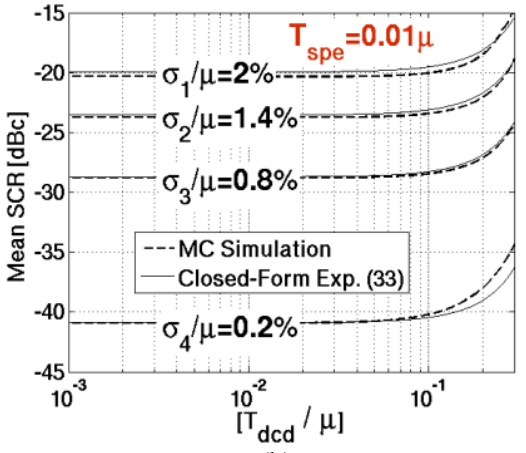

(b)

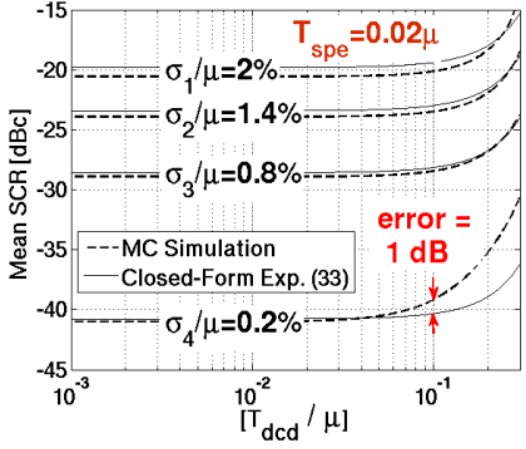

(c)

Fig. 9. Closed-form formula (33) vs. MC of (15) for synthesizer's mean SCR as a function of normalized DCD: (a) $\mathrm{T}_{\mathrm{spe}}=0$, (b) $\mathrm{T}_{\mathrm{spe}}=1 \mathrm{ps}$, and (c) $\mathrm{T}_{\mathrm{spe}}=2 \mathrm{ps}$.

ratio distribution of Rayleigh random variable $S_{n}$ over the random variable $C$, i.e. the magnitude of the fundamental tone. Fourier coefficients of $C$, i.e., $a_{N}$ and $b_{N}$, are calculated from (11) and (13) respectively, for $n=N$. In order to characterize the ratio random variable $\mathrm{SCR}$, we depict the mean and variance of $S_{n}$ and $C$ as functions of delay SD in Fig. 8(a). It can be observed that the mean of the fundamental tone $E[C]$, is much larger than its own variance $\operatorname{var}[\mathrm{C}]$, as well as the mean $E[S]$ and variance $\operatorname{var}[S]$ of the numerator $S_{n}$. Therefore, we approximate the SCR distribution by replacing $C$ with its mean, and modify (15) as

$$
S C R_{n}=S_{n} / E[C]
$$

To find $E[C]$, we plot the carrier coefficients $a_{N}$ and $b_{N}$ in Fig. 8(b) and observe that the mean values $E\left[a_{N}\right]$ and $E\left[b_{N}\right]$, are much larger than the variances $\operatorname{var}\left[a_{N}\right]$ and $\operatorname{var}\left[b_{N}\right]$. So, we approximate $E[C]$ as

$$
E[C]=E\left[\sqrt{{a_{N}{ }^{2}+b_{N}{ }^{2}}^{2}}\right] \approx \sqrt{\left(E\left[a_{N}\right]\right)^{2}+\left(E\left[b_{N}\right]\right)^{2}} .
$$

Shown in Fig. 8(b), the approximated $E[C]$ closely matches its MC-simulated counterpart. For small delay SD values, (30) can be further simplified to

$$
E[C] \approx \frac{2}{N \pi}\left|\frac{\sin \left(k N \pi / T_{r e f}\right) \sin \left(T_{s p e} N \pi / T_{r e f}\right)}{\sin \left(T_{s p e} \pi / T_{r e f}\right)}\right|, T_{s p e} \neq 0
$$

Finally, as SCR is the ratio of Rayleigh variable $S_{n}$ to a constant, we use linearity to approximate SCR also as a Rayleigh variable with PDF and mean of

$$
p_{X}(x)= \begin{cases}\left(x / \sigma_{R}^{2}\right) \exp \left(-x^{2} / 2 \sigma_{R}^{2}\right) & x \geq 0 \\ 0 & x<0\end{cases}
$$

$$
E\left[S C R_{n}\right]=\sigma_{R} \sqrt{\pi / 2}
$$

where

$$
\sigma_{R}=\sigma_{c o f} / E[C]
$$

Logarithmic representation of the PDF in (32) is obtained by change of variables, resulting in Log-Rayleigh PDF [14] of

$$
p_{Y}(y)=\left(10^{\frac{y}{10}} / \sigma_{R}^{2}\right) \exp \left(-10^{\frac{y}{10}} / 2 \sigma_{R}^{2}\right) \text {. }
$$

To verify the analysis, the distribution of the synthesizer's SCR is illustrated in Fig. 8(c). The dashed curve shows the normalized closed-form SCR PDF of (35), and the bar chart is the SCR histogram obtained from MC of our analytical model.

\section{Closed-Form Expression Accuracy}

In this part we evaluate the accuracy of the proposed Rayleigh-approximated closed-from formula, for large variations in SPE, DCD, and delay SD. To do so, we consider four different normalized delay SD values of $\sigma / \mu=0.2 \%$, $0.8 \%, 1.4 \%$, and $2 \%$, as well as three SPE values of $T_{\text {spe }}=0,1$, and 2 ps. Then we sweep DCD from $T_{d c d}=0.1$ to 30 ps. Fig. 9 plots the calculated mean SCR values from the closed-form expression (33) and compares them with the MC-simulated SCR of the analytical model (15). Shown in Fig. 9(a), with a zero SPE, the simulation and prediction follow each other even at a large DCD of 30 ps. A small offset of less than a $\mathrm{dB}$ is observable for the large delay SD of $\sigma / \mu=2 \%$, which is in line with our discussion in the previous part, and disappears as the delay SD gets smaller. On the other hand, as the DCD and SPE values get much larger compared to the delay $\mathrm{SD}$ value, the accuracy gets degraded. Analysis of several simulations 


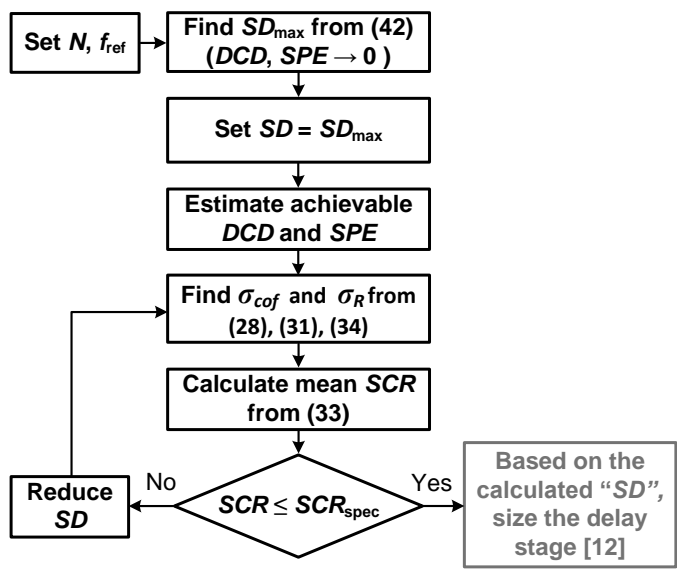

Fig. 10. Flowchart of the proposed spur-aware design flow.

indicates that at large DCD and SPE values for which

$$
\frac{T_{s p e}}{\mu} \cdot \frac{T_{d c d}}{\mu}>\frac{\sigma}{\mu},
$$

the criteria of (16) which is required for Rayleigh approximation, would not satisfy anymore. However, for reasonable values of SPE, DCD, and delay SD in the context of frequency synthesis, the proposed closed-form expression can predict the SCR of the largest spurs, with an acceptable accuracy. Fig. 9(c) demonstrates that a less than $1 \mathrm{~dB}$ error in SCR estimation can be obtained even for a small delay SD of $\sigma / \mu=0.2 \%$, and with reasonable DCD and SPE values of $T_{d c d}$ $=10 \mathrm{ps}$ and $T_{s p e}=2 \mathrm{ps}$, achievable in state-of-the-art CMOS implementations [2], [6], [15].

\section{A Spur-Aware Synthesizer Design Flow}

Based on our analysis in Section IV which led to a closefrom formula for the output SCR, we now propose a spuraware DLL-based frequency synthesizer design flow. This methodology determines the required value of the stage-delay $\mathrm{SD}$, at which a certain spurious performance (SCR) at synthesizer's output is achieved. Since the proposed design flow does not employ $\mathrm{MC}$ simulations, the overall iterative design process to find the required delay SD is accelerated. Prior to discussing the flow, we first derive an expression for the best SCR for a given delay SD, which is achieved when SPE and DCD are negligibly small. To do so, we first simplify the variance of the output spur Fourier coefficients $(n \neq N)$ which is given in (26) and (27). For $T_{s p e} \approx T_{d c d} \approx 0$, we have

$$
\begin{gathered}
\operatorname{var}\left[a_{n}\right]_{\min }=\frac{N\left(\sigma_{f}^{2}+\sigma_{r}^{2}\right)[2-\cos (2 n \pi / N)]}{2 T_{r e f}^{2} \sin ^{2}(n \pi / N)}, n \neq N \\
\operatorname{var}\left[b_{n}\right]_{\min }=\frac{N\left(\sigma_{f}^{2}+\sigma_{r}^{2}\right)[2+\cos (2 n \pi / N)]}{2 T_{r e f}^{2} \sin ^{2}(n \pi / N)}, n \neq N \\
\sigma_{c o f, \text { min }}^{2} \approx \frac{\operatorname{var}\left[a_{n}\right]_{\min }+\operatorname{var}\left[b_{n}\right]_{\min }}{2}=\frac{N\left(\sigma_{f}^{2}+\sigma_{r}^{2}\right)}{T_{r e f}^{2} \sin ^{2}(n \pi / N)}, n \neq N
\end{gathered}
$$

Also for very small SPE and DCD values, the mean magnitude of the carrier $(n=N)$ in (30) is simplified as

$$
E[C]=\frac{2 T_{r e f}^{2}-(N+1) N^{2} \pi^{2}\left(\sigma_{f}^{2}+\sigma_{r}^{2}\right)}{\pi T_{r e f}^{2}} \approx \frac{2}{\pi} .
$$

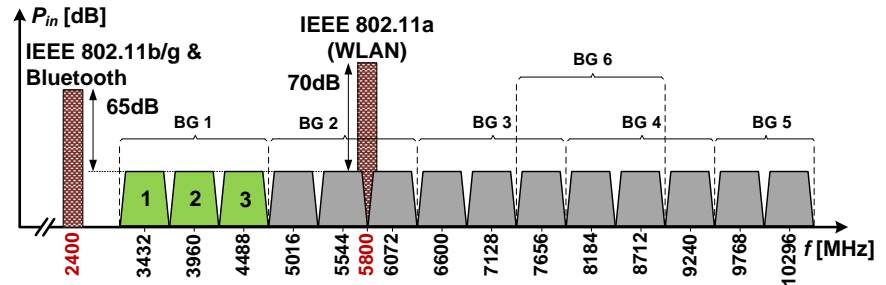

Fig. 11. WiMedia-UWB frequency allocation and the coexisting standards.

Now, by substitution of (39) and (40) into (33) and (34), the lower bound on the achievable mean SCR is determined as

$$
E\left[S C R_{n}\right] \geq \frac{\pi \sqrt{\pi}}{2 \sqrt{2 N} \sin (n \pi / N)} \times \frac{\sqrt{\sigma_{f}^{2}+\sigma_{r}^{2}}}{\mu} .
$$

The above expression indicates the best achievable spur suppression for a given delay mismatch and very small SPE and DCD values. Now, by knowing the spurious performance specification $\mathrm{SCR}_{\text {spec }}$ of the target standard, i.e. the maximum allowed spur-to-carrier level, we can reformulate (41) to find an upper bound on the normalized delay $\mathrm{SD}$, as

$$
\frac{\sqrt{\sigma_{f}^{2}+\sigma_{r}^{2}}}{\mu} \leq S C R_{\text {spec }} \times \frac{2 \sqrt{2 N}|\sin (n \pi / N)|}{\pi \sqrt{\pi}} .
$$

The proposed spur-aware methodology is illustrated in form of a flow chart in Fig. 10. First, from the specifications of the target wireless standard, which provides information such as the frequency bands, channel spacing, and switching time, we determine the design parameters including the reference clock frequency $f_{\text {ref }}$, and the number of delay stages $N$. Then from (42) we find the upper bound on the delay $\mathrm{SD}, \mathrm{SD}_{\max }$, by which a given $\mathrm{SCR}_{\text {spec }}$ satisfies when SPE and DCD are sufficiently small. Afterwards, to consider the effect of nonzero SPE and DCD, we use the initially-calculated $\mathrm{SD}_{\max }$ as well as the estimated achievable SPE and DCD values, to calculate $\sigma_{c o f}$ from (28), $E[C]$ from (31), and finally $\sigma_{R}$ from (34). Now we can determine the provided mean SCR from (33). If it is larger than $\mathrm{SCR}_{\text {spec }}$, then we improve our initial delay SD and calculate the new mean SCR, and repeat this procedure until $\mathrm{SCR} \leq \mathrm{SCR}_{\text {spec}}$. Based on the calculated value of the required delay $\mathrm{SD}$, one can do the sizing of the delay stage transistors using a similar approach as in [12].

\section{WiMEDIA-UWB SYNTHESIZER; A CASE STUDY}

In this section we employ the proposed design flow in Section V to find the required stage-delay SD for a DLL-based frequency synthesizer, which satisfies the spurious performance requirement of WiMedia-UWB bandgroup (BG) 1 standard [16].

\section{A. Design Procedure}

The spectrum allocation of the standard as well as the coexisting wireless technologies is illustrated in Fig. 11. The spur suppression requirement of WiMedia-UWB is calculated from the interferer scenario [17]. As can be seen in Fig. 11, because of the strong out-of-band interferers from IEEE $802.11 \mathrm{a} / \mathrm{b} / \mathrm{g}$, those spurious tones which fall within 2.4 to 5 $\mathrm{GHz}$ range, should maintain below $-45 \mathrm{dBc}$ [17], [18]. Note 


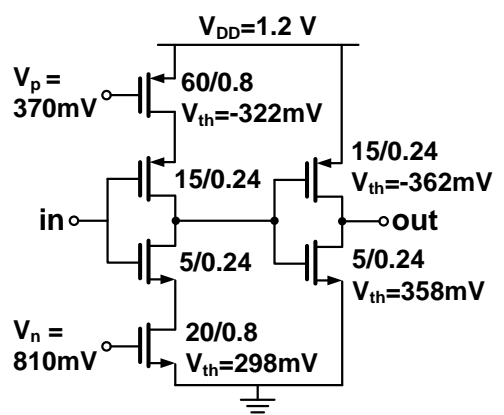

Fig. 12. Delay stage; a current-starved inverter with output buffer.

that the SCR provided by the DLL can be relaxed by a few $\mathrm{dBs}$ depending on the synthesizer architecture. The V-I converter-based EC with LC tank load [1] will band-pass-filter the spurs to some extent, depending on its $Q$ factor. In addition, utilizing as twice as the required input frequency and performing frequency division to generate quadrature carriers, will also suppress the spur levels. Therefore, we assume a $6 \mathrm{~dB}$ of suppression is already provided, implying that $\mathrm{SCR}_{\text {spec }}$ of $39 \mathrm{dBc}$ should be delivered. Shown in Fig. 11, WiMedia BG 1 consists of three sub-bands with center frequencies at $264 \times 13$, $264 \times 15$, and $264 \times 17 \mathrm{MHz}$, respectively. Hence, sub-band 3 has the maximum number of delay stages $N=17$. Moreover, a reference clock of $f_{\text {ref }}=2 \times 264 \mathrm{MHz}$ is utilized. Following the proposed flow, we calculate $\mathrm{SD}_{\max }$ from (42) which provides $\mathrm{SCR}_{\text {spec }}=-39 \mathrm{dBc}$ for the largest harmonic spur $(n=16)$,

$$
\left(\frac{\sqrt{\sigma_{f}^{2}+\sigma_{r}^{2}}}{\mu}\right)_{\max }=10^{\frac{-39}{20}} \frac{2 \sqrt{34}|\sin (16 \pi / 17)|}{\pi \sqrt{\pi}}=0.43 \% .
$$

We now use this value along with our estimated achievable values of SPE and DCD, i.e. $T_{s p e}=0.02 \mu \approx 2 \mathrm{ps}$ and $T_{d c d}=0.1 \mu$ $\approx 11 \mathrm{ps}$, respectively, to calculate the provided mean spur suppression from (33), as

$$
E\left[S C R_{16,1}\right]_{d B}=20 \log \left(\sigma_{R, 1} \sqrt{\pi / 2}\right) \approx-38 \mathrm{dBc} .
$$

Since this value is larger than $\mathrm{SCR}_{\text {spec }}$, we reduce the normalized delay SD from $0.43 \%$ to $0.4 \%$ (e.g., $\sigma_{r}=\sigma_{f}=0.31$ ps) and calculate the new SCR as

$$
E\left[S C R_{16,2}\right]_{d B}=20 \log \left(\sigma_{R, 2} \sqrt{\pi / 2}\right) \approx-39 \mathrm{dBc} .
$$

Consequently, we have determined the value of delay SD, for which the required $\mathrm{SCR}_{\text {spec }}$ by WiMedia-UWB is satisfied.

Now by knowing the required delay SD, transistor sizing of the delay stage circuit is straightforward. The mismatch characteristic of MOS transistors is a well-studied subject. The standard deviation of the drain current $I_{d}$ and threshold voltage $V_{t h}$ due to the device mismatch is described by the following equations [10],

$$
\begin{gathered}
\frac{\sigma_{I_{d}}}{I_{d}}=\frac{A_{\beta}}{\sqrt{W L}}+\frac{2 A_{V_{t h}}}{\left(V_{G S}-V_{t h}\right) \sqrt{W L}} \\
\sigma_{V_{t h}}=\frac{A_{V_{l h}}}{\sqrt{W L}}
\end{gathered}
$$

where $W L$ is the device area, and $A_{\beta}$ and $A_{V t h}$ are the technology-dependent constants. The above equations indicate that increasing the overdrive voltage will reduce the deviation of $I_{d}$, and increasing the transistor area $W L$ limits the deviation

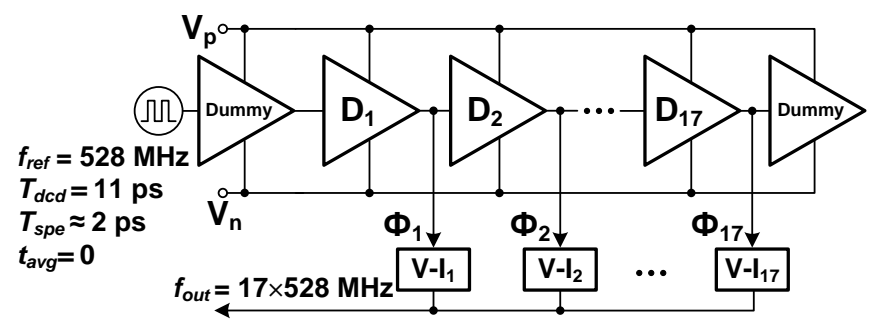

Fig. 13. Simulated testbench of WiMedia-UWB synthesizer. Due to openloop operation, $t_{a v g}=0$, and $T_{\text {spe }}$ may slightly deviate from $2 \mathrm{ps}$ for each MC sample.

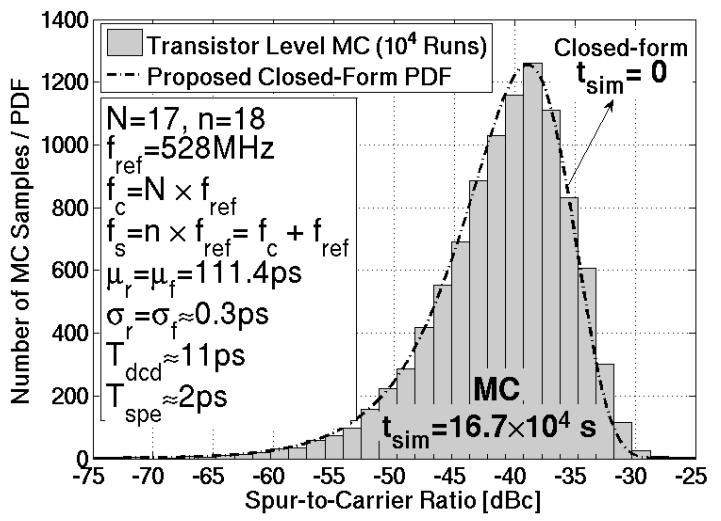

Fig. 14. SCR distribution at WiMedia-UWB synthesizer output spectrum, for the harmonic spur at $f_{s}=f_{\mathrm{c}}+f_{\text {ref. }}$.

of both $V_{t h}$ and $I_{d}$. An analytic model of the delay stage similar to that in [12] can be developed to estimate the required transistor areas. In order to provide the required matching with optimum device areas, we have utilized low threshold voltage (LVT) devices to increase the overdrive voltages. The transistor sizing and biasing details regarding a standard 65$\mathrm{nm}$ CMOS implementation of a current-starved delay stage which provides the calculated normalized delay SD of $\sqrt{\sigma_{f}^{2}+\sigma_{r}^{2}} / \mu=0.4 \%$, are indicated in Fig. 12. Note that to synthesize the carrier for all three sub-bands of BG 1, the number of delay stages in VCDL should be reconfigurable [4], [19] among $N=13,15$, and 17. This implies that although $N$ decreases when the synthesizer switches into the lower subbands, the control voltage also drops. The voltage drop value depends on VCDL voltage-to-delay transfer function. Hence, the SCR specification of the synthesizer puts a lower bound on the VCDL gain by indicating the minimum tolerable overdrive voltage at which $\mathrm{SCR}_{\text {spec }}$ also satisfies for sub-band 1 .

\section{B. Evaluation of the Results}

In this part we present the transistor-level MC simulation result regarding the spurious performance of the DLL-based WiMedia-UWB synthesizer, discussed in part A, and compare it with our analytical derivations. The simulated synthesizer testbench is depicted in Fig. 13. In order to reduce the MC simulation time, we replace the DLL with an open-loop VCDL of 17 current-starved delay stages of Fig. 12, while a constant loop control voltage is utilized. We also use 17 ideal V-I converters and short their outputs to perform edge-combining function and generate the multiplied carrier frequency $f_{c}=$ $17 \times 528 \mathrm{MHz}$. The MC simulation on the transistor-level 
testbench is performed using Spectre Cadence in a standard 65-nm CMOS process. To increase the simulator accuracy, we set the tolerance options as relative tolerance $=10^{-5}$, absolute voltage tolerance $=10^{-8}$, and absolute current tolerance $=10^{-}$ ${ }^{13}$. Also, in order to speed up the simulations, we enabled the accelerated parallel simulator (APS) and multithreading with 16 threads on 16 available processors. The bar chart in Fig. 14 show the transistor-level MC histogram of the WiMedia-UWB synthesizer's output SCR, for the reference harmonics at $f_{s}=f_{c}$ $+f_{\text {ref }}=9504 \mathrm{MHz}$. The dashed line represents the normalized predicted PDF of the WiMedia-UWB synthesizer's SCR, using the proposed closed-form formula of Log-Rayleigh approximation (35). Note that among those simulation parameters listed in Fig. 14, the assumed SPE value of $T_{\text {spe }}=2$ ps can be slightly different for the case of transistor-level MC simulation, as an open loop VCDL is utilized $\left(t_{\text {avg }}=0\right)$. With the above configurations and settings, the measured simulation time regarding a single design iteration using $10^{4} \mathrm{MC}$ samples on the open-loop VCDL is about $16.7 \times 10^{4} \mathrm{~s}$ (16.7 s/sample). Note that the simulation time becomes even higher when utilizing a closed-loop DLL for simulation. By employing the proposed methodology on the other hand, the design iterations are performed on the closed-form expressions (Fig. 10), where no simulation is involved. It can be observed from Fig. 14 that the predicted SCR PDF regarding the largest out-of-band harmonic spur at DLL-based WiMedia-UWB synthesizer output spectrum follows the corresponding MC histogram. Also the required $\mathrm{SCR}_{\text {spec }}$ of $-39 \mathrm{dBc}$ is satisfied and the predicted and simulated mean SCR values are closely matched with less than $1 \mathrm{~dB}$ error. Considering the utilized values of DCD, SPE, and delay SD, this result is in line with the accuracy condition in (36).

\section{CONCLUSION}

Fixed-pattern jitter in DLL-based edge-combing frequency synthesizers is generated due to phase misalignment of the DLL output edges. The resulting harmonic spurs down-convert the out-of-band interferes into the wanted channels, corrupting the desired signal. This paper proposes a comprehensive behavioral model for DLL-based frequency synthesizers which includes the effect of delay mismatch, SPE, and DCD on synthesizer spurious performance. From the proposed model and employing the Fourier series analysis of the DLL output edges, an analytical model is obtained which formulates the spur-to-carrier ratio SCR, at synthesizer's output. The analytical expression is further developed by employing statistical methods, to derive a close-form expression for the harmonic SCR. Based on our closed-form derivations, a spuraware design flow is introduced which determines the required stage-delay SD for a given SCR performance without using MC simulations, which speeds up the overall iterative design procedure. As an experimental case, the proposed design methodology is utilized to calculate the stage-delay SD of a DLL-based synthesizer, at which the SCR of WiMedia-UWB standard is satisfied. Based on the calculated delay SD, the delay stage is then designed in a standard 65-nm CMOS process. Transistor-level MC simulation regarding the synthesizer's spurious performance demonstrates good compliance with behavioral model and analytical predictions.

\section{APPENDIX A}

The Fourier series of the $m^{\text {th }}$ DLL output phase (neglecting the DC component) is expressed as

$$
\Phi_{m}(t)=\sum_{n=1}^{\infty} a_{n, \Phi_{m}} \cos \left(n \omega_{r e f} t\right)+\sum_{n=1}^{\infty} b_{n, \Phi_{m}} \sin \left(n \omega_{r e f} t\right) .
$$

From the waveform representation of Fig. 2, the Fourier coefficients $a_{n, \Phi}$ and $b_{n, \Phi m}$ in (48) are expressed as

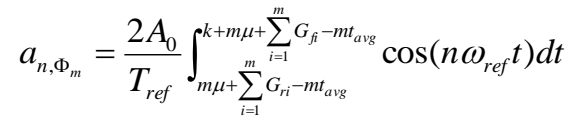

$$
\begin{aligned}
& b_{n, \Phi_{m}}=\frac{2 A_{0}}{T_{r e f}} \int_{m \mu+}^{k+m \mu+\sum_{i=1}^{m} G_{r i}^{m} G_{r i t}^{m}-m t_{\text {avg }}} \sin \left(n \omega_{r e f} t\right) d t .
\end{aligned}
$$

Since the EC output is constructed by summation of the multiphase DLL signals, its Fourier series can be written as

$$
\begin{aligned}
& \Phi_{\text {out }}(t)=\sum_{m=1}^{N} \Phi_{m}(t) \\
& =\sum_{m=1}^{N} \sum_{n=1}^{\infty} a_{n, \Phi_{m}} \cos \left(n \omega_{r e f} t\right)+\sum_{m=1}^{N} \sum_{n=1}^{\infty} b_{n, \Phi_{m}} \sin \left(n \omega_{r e f} t\right) \\
& =\sum_{n=1}^{\infty}\left(\cos \left(n \omega_{r e f} t\right) \sum_{m=1}^{N} a_{n, \Phi_{m}}\right)+\sum_{n=1}^{\infty}\left(\sin \left(n \omega_{r e f} t\right) \sum_{m=1}^{N} b_{n, \Phi_{m}}\right)
\end{aligned}
$$

Implied by linearity in (51), Fourier coefficients of the EC output are the sum of Fourier coefficients of the DLL phases,

$$
a_{n}=\sum_{m=1}^{N} a_{n, \Phi_{m}}, b_{n}=\sum_{m=1}^{N} b_{n, \Phi_{m}}
$$

\section{APPENDIX B}

Applying Taylor series approximation to (20), we get

$$
\begin{gathered}
a_{n}=A_{n} \sum_{m=1}^{N}\left[\alpha_{m} \kappa_{s}-\alpha_{m} \kappa_{s} \lambda^{2}\left(X_{f m}^{2}+X_{r m}^{2}\right)+\alpha_{m} \kappa_{c} \lambda\left(X_{f m}-X_{r m}\right)\right. \\
\left.-\beta_{m} \kappa_{s} \lambda\left(X_{f m}+X_{r m}\right)-\beta_{m} \kappa_{c} \lambda^{2}\left(X_{f m}^{2}-X_{r m}^{2}\right)\right] .
\end{gathered}
$$

The mean of $a_{n}$ is derived from its first moment as

$$
\begin{aligned}
E\left[a_{n}\right]=A_{n} \sum_{m=1}^{N} & \left(\alpha_{m} \kappa_{s}-\alpha_{m} \kappa_{s} \lambda^{2} E\left[X_{f m}^{2}+X_{r m}^{2}\right]\right. \\
& +\alpha_{m} \kappa_{c} \lambda E\left[X_{f m}-X_{r m}\right]-\beta_{m} \kappa_{s} \lambda E\left[X_{f m}+X_{r m}\right] \\
& \left.-\beta_{m} \kappa_{c} \lambda^{2} E\left[X_{f m}^{2}-X_{r m}^{2}\right]\right) .
\end{aligned}
$$

To calculate $E\left[a_{n}\right]$, we first utilize (12) to write

$$
\begin{gathered}
E\left[X_{r m}\right]=E\left[X_{r m}\right]=0 \\
E\left[X_{r m}^{2}\right]=m\left(1-\frac{m}{N}\right) \sigma_{r}^{2}, E\left[X_{f m}^{2}\right]=m\left(\sigma_{f}^{2}+\frac{m}{N} \sigma_{r}^{2}\right) .
\end{gathered}
$$

Then from linearity, we calculate the required expectations as

$$
\begin{gathered}
E\left[X_{f m}-X_{r m}\right]=E\left[X_{f m}+X_{r m}\right]=0 \\
E\left[X_{f m}^{2}+X_{r m}^{2}\right]=m\left(\sigma_{f}^{2}+\sigma_{r}^{2}\right) \\
E\left[X_{f m}^{2}-X_{r m}^{2}\right]=m\left(\sigma_{f}^{2}+\sigma_{r}^{2}\left(\frac{2 m}{N}-1\right)\right) .
\end{gathered}
$$


To obtain $a_{n}$ variance, $\left(E\left[a_{n}\right]\right)^{2}$ and $E\left[a_{n}^{2}\right]$ are required. The former is determined from (54). The latter is derived by calculating $a_{n}^{2}$ from (53), and then applying the expectation. Substituting the resulting approximated expressions into (17),

$$
\operatorname{var}\left[a_{n}\right] \approx A_{n}^{2} \lambda^{2}\left\{\kappa_{c}^{2} A+\kappa_{s}^{2} B-2 \kappa_{s} \kappa_{c} C\right\}
$$

where

$$
\begin{gathered}
A=E\left[\left(\sum_{m=1}^{N} \alpha_{m}\left(X_{f m}-X_{r m}\right)\right)^{2}\right]=\left(\sigma_{f}^{2}+\sigma_{r}^{2}\right) \\
\times\left(\sum_{m=1}^{N} m \alpha_{m}^{2}+2 \sum_{m=1}^{N-1}\left(m \alpha_{m} \sum_{i=m+1}^{N} \alpha_{i}\right)\right) \\
B=E\left[\left(\sum_{m=1}^{N} \beta_{m}\left(X_{f m}+X_{r m}\right)\right)^{2}\right]=\left(\sigma_{f}^{2}+\sigma_{r}^{2}\right) \\
\times\left(\sum_{m=1}^{N} m \beta_{m}^{2}+2 \sum_{m=1}^{N-1}\left(m \beta_{m} \sum_{i=m+1}^{N} \beta_{i}\right)\right) \\
C=E\left[\sum_{m=1}^{N} \alpha_{m}\left(X_{f m}-X_{r m}\right) \sum_{m=1}^{N} \beta_{m}\left(X_{f m}+X_{r m}\right)\right]=\sum_{m=1}^{N} \alpha_{m}\left(\sum_{i=1}^{m} i \beta_{i}\right. \\
\left.\left[\sigma_{f}^{2}+\left(\frac{2 m}{N}-1\right) \sigma_{r}^{2}\right]+m \sum_{i=m+1}^{N} \beta_{i}\left[\sigma_{f}^{2}+\left(\frac{2 i}{N}-1\right) \sigma_{r}^{2}\right]\right)
\end{gathered}
$$

\section{REFERENCES}

[1] G. Chien and P. R. Gray, "A 900-MHz Local Oscillator using a DLLBased Frequency Multiplier Technique for PCS Applications," IEEE J. Solid State Circuits, vol. 35, no. 12, pp. 1996-1999, Dec. 2000.

[2] J. Zhuang, Q. Du, and T. Kwasniewski, "A $-107 \mathrm{dBc} 10 \mathrm{kHz}$ carrier offset 2-GHz DLL-based frequency synthesizer," Proc. IEEE Custom Integr. Circuits Conf., pp. 301-304, Sep. 2003.

[3] T. C. Lee and K. J. Hsiao, "A DLL-based frequency multiplier for MBOA-UWB system," Proc. Symp. VLSI Circuits Dig. Tech. Papers, pp. 42-45, Jun. 2005.

[4] T. C. Lee and K. J. Hsiao, "The Design and Analysis of a DLL-Based Frequency Synthesizer for UWB Application," IEEE J. Solid State Circuits, vol. 41, no. 6, pp. 1245-1252, Jun. 2006.

[5] S. Gierkink, "An $800 \mathrm{MHz}-122 \mathrm{dBc} / \mathrm{Hz}-a t-200 \mathrm{kHz}$ clock multiplier based on a combination of PLL and recirculating DLL," Proc. IEEE Int. Solid-State Circuits Conf., pp. 454-455, Feb 2008.

[6] P. C. Maulik and D. A. Mercer, "A DLL-based programmable clock multiplier in $0.18 \mu \mathrm{m}$ CMOS with $-70 \mathrm{dBc}$ reference spur," IEEE J. Solid-State Circuits, vol. 42, no. 8, pp. 1642-1648, Aug. 2007.

[7] N. Khan, M. Hossain, K.L.E. Law, "A Low Power Frequency Synthesizer for 60-GHz Wireless Personal Area Networks," IEEE Trans. Circuits Syst. II: Exp. Briefs, vol.58, no.10, pp. 622-626, Oct. 2011.

[8] H. J. Ng et al., "A DLL-Supported, Low Phase Noise Fractional-N PLL with a Wideband VCO and a Highly Linear Frequency Ramp Generator for FMCW Radars," IEEE Trans. Circuits Syst. I: Reg. Papers, vol. 60, no. 12, pp. 3289-3302, Dec. 2013.

[9] A. Elshazly et al., "A $1.5 \mathrm{GHz} 890 \mathrm{uW}$ Digital MDLL with 400fsrms Integrated Jitter, $-55.6 \mathrm{dBc}$ Reference Spur and 20fs/mV Supply-Noise Sensitivity Using 1b TDC," Proc. IEEE Int. Solid-State Circuits Conf., Dig. Tech. Papers, pp. 242-244, Feb. 2012.

[10] M. J. M. Pelgrom, A. C. J. Duinmaijer, and A. P. G. Welbers, "Matching properties of MOS transistors," IEEE J. Solid-State Circuits, vol. 24, no. 10, pp. 1433-1440, Oct. 1989.

[11] J. Zhuang, Q. Du and T. Kwasniewski, "Noise, spur characteristics and in-lock error reduction of DLL-based frequency synthesizers," Proc. Int. Conf. Commun., Circuits Syst., pp. 1443-1446, Jun. 2004.

[12] O. Casha et al., "Analysis of the Spur Characteristics of EdgeCombining DLL-Based Frequency Multipliers," IEEE Trans. Circuits Syst. II: Exp. Briefs, vol. 56, no. 2, pp. 132-136, Feb. 2009.
[13] J.G. Proakis, and M. Salehi, Digital Communications, $5^{\text {nd }}$ Edition, McGraw Hill, 2008.

[14] B. Rivet, L. Girin and C. Jutten, "Log-Rayleigh Distribution: A Simple and Efficient Statistical Representation of Log-Spectral Coefficients," IEEE Trans. Audio, Speech, Lang. Process., vol. 15, no. 3, pp. 796802, Mar. 2007.

[15] T. Ali, et al., "A 4.6GHz MDLL with $-46 \mathrm{dBc}$ reference spur and aperture position tuning," Proc. IEEE Int. Solid-State Circuits Conf. Dig. Tech. Papers, pp. 466-467, 2011.

[16] High rate Ultra Wideband PHY and MAC Standard, ECMA-368. $3^{\text {rd }}$ Edition, Dec. 2008 [Online].

[17] K. Iniewski, Wireless Technologies: Circuits, Systems, and Devices. New York: CRC Press, 2007.

[18] M. M. Izad and C. Hng "A Pulse Shaping Technique for Spur Suppression in Injection-Locked Synthesizers," IEEE J. Solid State Circuits, vol. 47, no. 3, pp. 652-664, Mar. 2012.

[19] A. Ojani, B. Mesgarzadeh, and A. Alvandpour, "A DLL-Based Injection-Locked Frequency Synthesizer for WiMedia UWB," Proc. IEEE Int. Symposium Circuits Syst., pp. 2027-2030, May 2012.

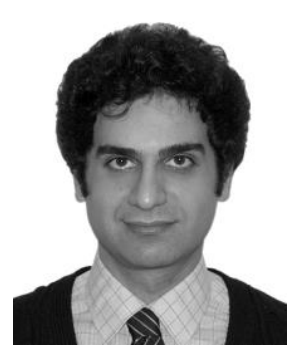

Amin Ojani (S'10) received the M.Sc. degree in electrical engineering from Linköping University, Linköping, Sweden, in 2008. He is currently working towards the Ph.D. degree at Linköping University.

Between 2008 and 2009, he was with Ericsson $\mathrm{AB}$, Lund, Sweden, where he worked on clock generation and distribution for Ericsson's mobile platforms. His research interests include phaselocked systems and RF synthesizers.

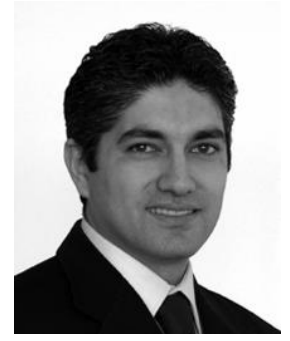

Behzad Mesgarzadeh (S'02-M'09) received the B.Sc. degree in electrical engineering from Sharif University of Technology, Tehran, Iran, in 2000, and the M.Sc. and Ph.D. degrees in electrical engineering from Linköping University, Linköping, Sweden, in 2004 and 2008, respectively.

$\mathrm{He}$ is currently an Assistant Professor at the Department of Electrical Engineering, Linköping University. His research interests include lowpower clocking techniques, clock generators and frequency synthesizers, and high-data-rate wireless communication systems.

Dr. Mesgarzadeh was the recipient of the 50th IEEE Midwest Symposium on Circuits and Systems Best Student Paper Award in 2007.

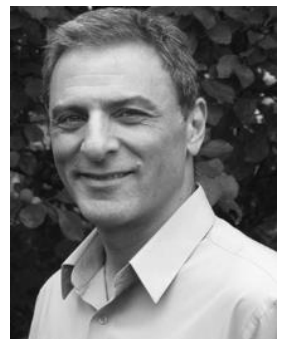

Atila Alvandpour (M'99-SM'04) received the M.S. and Ph.D. degrees from Linköping University, Sweden, in 1995 and 1999, respectively.

From 1999 to 2003, he was a senior research scientist with Circuit Research Lab, Intel Corporation. In 2003, he joined the department of Electrical Engineering, Linköping University, as a Professor of VLSI design. Since 2004, he is the head of Electronic Devices division. His research interests include various issues in design of integrated circuits and systems in advanced nanoscale technologies, with a special focus on efficient data converters, wireless transceiver front-ends, onchip clock generators and synthesizers, sensor interface electronics, lowpower/high-performance digital circuits and memories, and chip design techniques. He has published more than 100 papers in international journals and conferences, and holds 24 U.S. patents.

Prof. Alvandpour is a senior member of IEEE, and has served on many technical program committees of IEEE and other international conferences, including the IEEE Solid-State Circuits Conference (ISSCC), and European Solid-State Circuits Conference (ESSCIRC). He has also served as guest editor for IEEE JOURNAL OF SOLID-STATE CIRCUITS. 